\title{
Herramientas para una niñez libre de violencias
}

\author{
La experiencia del proyecto de extensión universitaria \\ «Crianza con derechos» \\ Tools for a Violence-Free Childhood \\ The Experience of the University Extension Project «Parenting with Rights»
}

\author{
María Eugenia Labrunée \\ melabrun@mdp.edu.ar \\ https://orcid.org/0000-0002-7628-0446 \\ Universidad Nacional de Mar del Plata | Argentina
}

\section{Resumen}

La autora describe y analiza en este artículo las acciones llevadas a cabo en el marco del proyecto de extensión de la Universidad Nacional de Mar del Plata (UNMDP) «Crianza con derechos. Construcción de herramientas para una niñez libre de violencias en el hogar, las aulas y la atención sanitaria». El trabajo con familiares, con docentes y con profesionales de programas sociales en el oeste y norte de la ciudad de Mar del Plata (Buenos Aires, Argentina), acompañó la construcción de prácticas de crianza fundadas en el buen trato, a partir del fomento de espacios de reflexión y del desarrollo de herramientas para el apoyo y la promoción de la crianza con derechos.

\section{Palabras clave}

crianza, derechos, cuidado, niñez

\begin{abstract}
The authoress describes and analyzes in this article the actions carried out in the framework of the extension (or Social Action) project of the Universidad Nacional de Mar del Plata (UNMDP) «Parenting with Rights: Building Tools for a Violence-Free Childhood at Home, Classrooms, and Health Care». The work with relatives, teachers and professionals of social programs in the west and north of Mar del Plata city (Buenos Aires, Argentina), accompanied the construction of parenting practices based on good treatment, from the promotion of fostered spaces for reflection and developed tools to support and promote parenting with rights.
\end{abstract}

\section{Keywords}

parenting, rights, care, childhood 


\section{Introducción}

En este artículo se describe y se analiza el camino recorrido por el equipo de trabajo del proyecto de extensión universitaria «Crianza con derechos. Construcción de herramientas para una niñez libre de violencias en el hogar, las aulas y la atención sanitaria», desarrollado durante 2019 e inscripto en la Universidad Nacional de Mar del Plata (UNMDP), Buenos Aires, Argentina. Mediante su intervención, se discute acerca de la existencia de una «crisis de los cuidados» (Pérez Orosco, 2006; Ezquerra, 2010) y se advierte sobre la distribución de tareas y las condiciones en las que se desarrolla el cuidado -tanto remunerado como no remunerado-, así como sobre las desigualdades de género que derivan de la carga de la mayor parte del trabajo social de cuidado sobre las mujeres. Se sitúa, además, en las implicancias de esta crisis para los sujetos de cuidado -niñes, en este caso-y para las políticas públicas específicas.

El objetivo del proyecto fue co-construir, junto con las instituciones y la comunidad, prácticas de crianza y de enseñanza fundadas en el buen trato, mediante un abordaje integral e interdisciplinario. El proyecto consideró aportes de la psicología del desarrollo, especialmente, la teoría del apego (Bowlby, 1988) y el psicoanálisis vincular. A su vez, se propuso trascender mediante el diseño conjunto de herramientas comunicativas y formativas que permitieron continuar las acciones una vez finalizado el año de ejecución. Las comunidades acompañadas por el proyecto fueron los barrios Las Heras, Coronel Dorrego, Malvinas Argentinas, Jorge Newbery y Camet Norte, de la ciudad argentina de Mar del Plata, cabecera del Partido de General Pueyrredon, provincia de Buenos Aires. Se ofrecieron espacios reflexivos y propositivos entre les adultes vinculades con niñes y con adolescentes (NNA), siempre bajo la premisa de favorecer prácticas respetuosas de crianza y de cuidado.

El trabajo que aquí se presenta se organiza de la siguiente manera: inicialmente, se describe el territorio en el cual se llevó adelante el proyecto. Luego, se ofrece la fundamentación conceptual sobre la cual se basó la estrategia desarrollada, para luego dar cuenta del abordaje interdisciplinario, en virtud de la formación y la expertise de las integrantes del equipo extensionista y el diálogo con las instituciones participantes. Con este background inicial, se exponen los fundamentos metodológicos considerados para el abordaje y los dispositivos diseñados, bajo la restricción de los recursos para llevarlos adelante. Finalmente, se describen de forma detallada las actividades y los resultados obtenidos, los diferentes talleres de reflexión-capacitación, las reuniones y las actividades de difusión para acompañar una crianza con derechos. Se exponen, también, 
las evaluaciones sobre lo realizado, obtenidas mediante la aplicación de encuestas a organizaciones vinculadas con el proyecto, que permitieron rescatar, además, nuevos intereses y preocupaciones en referencia al tema. Las reflexiones finales resaltan la importancia y la potencialidad del diálogo para reconocer y para revisar prácticas de crianza, establecer nuevos desafíos y vehiculizar instancias de intervención superadoras que trasciendan el impulso inicial.

\section{Características del territorio bajo intervención}

Mar del Plata es una ciudad costera con una población estable de 650.000 habitantes y líder en el desarrollo regional de su zona, el sudeste de la Provincia de Buenos Aires. Según los datos del Censo 2010, hay una importante presencia de personas menores de 20 años (30\%) y una distribución por sexo que favorece a las mujeres, con 52,2\%. $\mathrm{Su}$ estructura productiva está fuertemente basada en los servicios y marcada por una fuerte estacionalidad, al ser una ciudad visitada por turistas en el período estival. También se desarrolla un diversificado sector secundario, en el que resalta la actividad de la construcción y una industria consolidada, sobre todo de productos pesqueros -por situarse, allí, uno de los puertos más importantes del país- y de otros alimentos, así como la metalurgia. Entre las producciones primarias, además de las capturas pesqueras, existe un importante cordón periurbano frutihortícola.

El proyecto se desarrolló en el cordón suroeste y norte de la ciudad de Mar del Plata, áreas que han mostrado en los períodos 1990-2001 y 2001-2010 un alto crecimiento, aunque en el segundo caso a un ritmo menor, específicamente a nivel intraejidal, presionando hacia los bordes de la ciudad y trascendiendo la periferia inmediata.

Los barrios alcanzados por el proyecto presentan un incremento poblacional en el período 1991-2001, pertenecen a los bordes intraurbanos y periurbanos, tienen una acentuada tendencia a condiciones críticas, una alta vulnerabilidad social -debido a condiciones de un habitat-habitar no resueltas- y, además, una marcada participación de jóvenes y niñes (Sagua \& Sabuda, 2015).

Particularmente, en los barrios de la zona norte (Camet, Dalias y Parque Peña), la accesibilidad a agua potable y cloacas es reducida. En el trabajo de Virginia Maté (2017), realizado en el marco del Área Programática del Centro de Salud Municipal de Alto Camet, se especifican algunas características de estos barrios. Se expone que la zona de mayor vulnerabilidad socioeconómica y habitacional se ubica en dos grandes localizaciones: 
las llamadas «chancherías» y la zona próxima al aeroparque. Sobre la primera, se indica que presenta una situación particular debido a que las condiciones de habitabilidad de la mayoría de las viviendas son muy precarias (construidas con chapa, cartón y madera) y cuentan con escasos servicios. Se resalta que la población -de composición joven en los bordes externos- cuenta con una importante entrada de otras localidades y de países limítrofes, y se agrega que en el área programática se observa mucha migración interna: familias que se mudan dentro de la ciudad misma; familias que provienen de otras provincias (Santa Fe, Mendoza, Gran Buenos Aires, Ciudad de Buenos Aires, etc.); y familias provenientes de otros países de América Latina (Bolivia, Perú y Paraguay). Esta situación puede incidir en una escasa formación de lazos o de redes de contacto de los cuales las familias puedan hacer uso ante dificultades en la organización familiar.

Otro diagnóstico sobre la zona oeste y sur de la ciudad reveló que la población considerada tenía un perfil definido en cuanto a los principales sectores de inserción laboral, en el cual el comercio, la pesca, la construcción y el servicio doméstico presentaban una gravitación dominante. Además, entre quienes declaraban no trabajar, $40 \%$ efectuaba alguna tarea en su vivienda por la que percibía un ingreso. En tanto, se relevó que en $12,5 \%$ de los hogares con NNA menores de 12 años estos se quedaban solos cuando los adultos salían a trabajar (Alegre y otros, 2011).

A través del trabajo de investigación de Paola Cabero (2018), se han observado las dificultades para identificar las problemáticas de violencia familiar, debido a lo cual muchos de los casos quedan sin abordarse.

\section{Fundamentación de la intervención}

El término «niños con llave», popularizado en los Estados Unidos en la década de 1980, (Hochschild, 2008), describe las situaciones de un creciente número de niñes que al regresar de la escuela permanecen en un hogar sin un adulto a cargo, una realidad que conlleva a discutir acerca de la existencia de una «crisis de los cuidados» (Pérez Orosco, 2006; Ezquerra, 2010). Este es un campo de estudio que advierte acerca de la distribución de tareas y de las condiciones en las que se desarrolla el cuidado -tanto remunerado como no remunerado-, así como sobre las desigualdades de género que se derivan de la carga de la mayor parte del trabajo social de cuidado sobre las mujeres. Tal vez a causa de la intensidad de dichas desigualdades, han quedado postergadas sus implicancias para los sujetos de cuidado -niñes, en este caso-y para las políticas públicas específicas. 
En la Argentina, estas postergaciones han redundado en una combinación de modelos de cuidado tradicional -con desigual distribución del trabajo remunerado y no remunerado, y con el cuidado a cargo de las mujeres- sin una red de políticas de cuidado adecuada, lo que afecta la calidad del cuidado y la provisión de bienestar a les niñes (Hochschild, 2008). A nivel más pragmático, la asociación entre bienestar de niñes y dedicación exclusiva de las mujeres-madres a su cuidado y a su crianza promueve un ideal inaccesible para la mayor parte de las familias, puesto que asume la existencia de un hogar conyugal donde el trabajador varón provee recursos económicos y protección social al conjunto del núcleo familiar. Frente esto, más de una cuarta parte de la población ocupada en el país carece de seguridad social, un tercio no cuenta con aportes jubilatorios, ni aguinaldo ni vacaciones pagas y cerca de $10 \%$ de la población económicamente activa se encuentra desocupada ${ }^{1}$ (INDEC, 2018). Aun así, la crianza exclusiva por parte de las mujeres-madres resulta ser un imperativo más que una elección, frente a la escasez de políticas públicas de cuidado y a una deficiente distribución de trabajo doméstico y de cuidado no remunerado. Por esto, la principal demanda en términos de políticas de cuidado es la promoción de la universalización de las instituciones de cuidado desde edades tempranas (para 2015, Eliana Aspiazu y Romina Cutuli rescataban que menos de 3\% de niñes menores de tres años accede a instituciones educativas).

Además, la provisión de cuidado de calidad y respetuoso de los derechos de la niñez requiere de un andamiaje más complejo y de un abordaje integral. De allí que resulta primordial el desarrollo de estrategias comunicativas que permitan la visibilización por parte de les profesionales intervinientes, y de los propios protagonistas, de las situaciones que colocan a NNA en situaciones de vulneración de sus derechos, sujetos a prácticas de cuidado humillantes e incapaces de favorecer el desarrollo autónomo, y que se asocian a la quietud y a la reflexión al castigo, prácticas que este proyecto procura desnaturalizar, porque subestiman a NNA como sujetos de derechos y alientan a ignorar sus expresiones desde los primeros días de vida.

Lamentablemente, muchas de estas prácticas son avaladas desde la industria editorial y los medios de comunicación masiva. Desde fines del siglo XIX, las políticas de maternidad (Nari, 2004) impusieron prácticas de crianza homogeneizantes y disciplinadoras, centradas en la necesidad de un espacio de socialización primaria que asegurara la función de «policía de las familias» (Donzelot, 1979). Al mismo tiempo, perviven prácticas de crianza y de educación violentas tanto en el espacio del hogar como en la escuela, aun cuando sean discutidos sus fundamentos teóricos. 
El proyecto cuestiona, también, aquellas modalidades de crianza presuntamente más avanzadas en términos de respeto a los derechos de NNA, promovidas por la pediatría psicológica a mediados del siglo $\mathrm{xX}$, que mantienen lógicas homogeneizadoras $\mathrm{y}$ heteronormativas (Rustoyburu, 2012). Algunas evitan los castigos y favorecen el estímulo positivo en forma de premios materiales y simbólicos, pero refuerzan las acciones mediante el reconocimiento material y simbólico de les adultes de referencia, sin dar relevancia a la comprensión del sentido de dichas acciones y, por lo tanto, sin favorecer la construcción del pensamiento autónomo y crítico. Se discuten, asimismo, aquellas propuestas más respetuosas pero que corren el riesgo de profundizar los procesos de maternalización de las mujeres, al redundar en una desigual distribución del trabajo doméstico y de cuidado no remunerado, atravesado por nuevas exigencias que promoverían el bienestar de NNA sobre la base de una sobrecarga de trabajo de cuidado, en especial, de las mujeres-madres. Estos discursos dialogan con aquellos que legitiman desigualdades de género, a partir de los fundamentos biológico-fisiológicos de la maternidad. Excluyen, así, la diversidad de formas de crianza y de cuidado donde estos lazos no están presentes, y legitiman en los espacios públicos y productivos las discriminaciones que aún pesan sobre las mujeres.

Todas estas miradas atraviesan las concepciones y las prácticas de crianza en la Argentina, como se refleja en la Encuesta sobre las condiciones de vida de la niñez y adolescencia (Ministerio de Desarrollo Social-UNICEF, 2013). Allí se destaca que casi $70 \%$ de los encuestados considera que NNA deben ser castigados, aunque menos del $4 \%$ considera los castigos físicos como opción. Existe un amplio consenso en torno al castigo no físico como práctica pedagógica, pero este enmarca una amplia gama de prácticas conductistas que impulsan estrategias para eludir el castigo, antes que para desarrollar procesos de decisión autónomos e informados. "Quitar privilegios», por ejemplo, que se declara como practicada por más de $50 \%$ de las familias, no hace explícita la frontera entre aquello que es un privilegio y lo que constituye un derecho. Los castigos físicos, en tanto, presentan una clara disonancia entre su legitimidad y su práctica. Si bien es minoritaria su aceptación, en 40,5\% de hogares encuestados se aplicó el castigo físico (UNICEF, 2018).

En la búsqueda de factores sociales o vinculados a las características de los hogares para explicar la utilización de prácticas de crianza que vulneran los derechos de NNA se termina por estigmatizar algunas cuestiones. Por un lado, una correlación positiva entre escolaridad y erradicación de la violencia, que en realidad solo advierte sobre la insuficiencia del tránsito por la educación formal para construir vínculos respetuosos de los derechos de la niñez -entre otras razones, porque las propias instituciones reproducen en parte esas prácticas violentas-. Por otro lado, la identificación de las prácticas violentas de crianza 
con los segmentos de menor nivel educativo ofrece un artificio de distanciamiento entre la población afectada por la problemática y la que la analiza. Las situaciones dolorosas son más factibles tanto de reconocer como de nombrar a partir de la mirada en tercera persona, excepto que sea posible expresar defensa frente a la vulnerabilidad (Theidon, 2004). Los datos nos aportarían un panorama mucho más amplio, en el que las desigualdades se refuerzan en clave interseccional de clase, etnia, género y también edad (Carli, 2002). En este sentido, destacamos la necesidad de incorporar dicha perspectiva a las problemáticas vinculadas a la niñez y, a su vez, sumar la condición etaria al concepto de interseccionalidad (Llobet, 2014).

Dado que no existen excepciones en el tránsito por la niñez, reconocer las prácticas vulneradoras de derechos es aceptar la identificación como sujeto vulnerado. Por ello el abordaje de la problemática requiere de un particular esfuerzo, tanto para construir una mirada positiva de nuestro horizonte de expectativas, como para vencer las resistencias que se construyen frente a la identificación de las violencias en primera persona.

Las concepciones acerca de la niñez atraviesan todos los vínculos entre adultes y NNA en espacios públicos y privados, y la escuela es uno de los escenarios donde suelen reproducirse las prácticas violentas. Esta es la razón por la cual este proyecto interviene en la formación de docentes, específicamente, en el Instituto de Formación Docente Manuel Belgrano. Se consideran, aquí, las circunstancias desfavorables propias de las características de la escolaridad obligatoria, que requieren de un esfuerzo emocional y formativo por parte de les profesionales de la educación para evitar reproducir o reforzar situaciones de vulneración vinculadas con deficiencias materiales, tendencias homogeneizadoras, alto número de estudiantes por docente, etc. En los estudios sobre violencia escolar suelen analizarse las vulneraciones intrageneracionales entre NNA y se critican las estructuras punitivas formales (Forero-Londoño, 2011). Pero ha sido menos atendida la multiplicidad de instancias no formales en las que las prácticas adultas vulneran a través del insulto, la desacreditación, la ridiculización, la obligación de sometimiento, sin que existan normas formales que lo permitan o, incluso, contraviniendo normas institucionales. Puede arriesgarse que la autoridad de la figura docente, en conjunción con las dificultades metodológicas para incluir la voz de NNA en la investigación, redunda en que estas experiencias carezcan de suficiente sistematización.

Ello se intensifica en el caso de les profesionales de la salud, de allí el involucramiento con un centro municipal de atención primaria de salud. La bibliografía expone que en este ámbito, donde la niñez constituye un factor añadido de vulneración para las intervenciones no consentidas y/o no informadas, se difunden prácticas que no devienen o que, incluso, 
contravienen el saber disciplinar, y que se reproducen como recomendaciones desde el lugar de la autoridad médica. Existe un amplio consenso en torno al rol de les profesionales médicos en la identificación de las vulneraciones en el ámbito de la familia o de la comunidad, pero pareciera que no se dimensiona la responsabilidad que les cabe a estes profesionales en la legitimación y en la reproducción, o no, de prácticas vulneradoras de derechos. En esta institución de la zona sur de la ciudad de Mar del Plata se advierte una vinculación con la comunidad centrada en la atención de la enfermedad antes que en el cuidado integral de la salud. Esto es reconocido desde su gestión y este proyecto pretendió reorientar las miradas o los abordajes de los/as profesionales hacia formas positivas y propositivas de abordar la crianza y el cuidado. ${ }^{2}$

\section{El equipo de trabajo caracterizado por la interdisciplina}

El equipo de trabajo cuenta con una amplia trayectoria en el abordaje de problemáticas vinculadas con el cuidado, desde diversas perspectivas disciplinares. La directora, Romina Cutuli, ha integrado el cuidado infantil en el abordaje de las interacciones entre el mundo del trabajo remunerado y no remunerado. Desde los estudios del trabajo con perspectiva de género, se ha identificado la manifestación de una «crisis de los cuidados», expresada en la diversificación de formas de organización familiar y de procesos de precarización y de flexibilización laboral que no han sido acompañados por políticas de cuidado adecuadas a estas nuevas necesidades.

Las investigaciones realizadas han incluido el impacto en la vida familiar y de niñes, en particular, y las condiciones de trabajo de las mujeres-madres. También se han investigado las políticas de cuidado nacionales en perspectiva comparada, a fin de construir un diagnóstico sobre los alcances y las limitaciones en materia de cuidado en nuestro país. Desde las labores de extensión, se han llevado a cabo diversas actividades vinculadas al cuidado, entre los que se destaca el desarrollo de un proyecto de extensión radicado en la FCEyS y un Curso de Universidad de Verano (UNMDP), dictado durante tres años consecutivos.

La codirectora, autora de este artículo, se ha especializado en la temática de trabajo infantil y derechos de la niñez, mediante el desarrollo de investigaciones en el marco del Grupo de Estudios del Trabajo de la FCEYs. Es miembro del Consejo Local de Promoción y Protección de los Derechos de los NNA y Jóvenes desde 2009; fue miembro de la Mesa Interinstitucional por la prevención y erradicación del trabajo infantil y protección del trabajo adolescente del Partido de General Pueyrredon, entre 2013 y 2017; y participa del Observatorio de Niñez del Consejo Local. 
Entre las integrantes se encuentran:

* Especialistas en violencia de género en el ámbito familiar.

- Una participante de equipos de abordaje territorial en ese tema.

- Una formadora de profesionales de nivel inicial.

- La coordinadora del Centro de Desarrollo Infantil del barrio

Jorge Newbery de la ciudad de Mar del Plata.

- Una especialista en educación maternal.

- Graduadas de la licenciatura en trabajo social (UNMDP) con tesis

sobre representaciones de género y familia.

- Una capacitadora en Educación Sexual Integral y coordinadora

de proyectos culturales.

- Estudiantes de la Escuela Superior de Medicina -algunas, con trayectoria

como doulas- y de la licenciatura en psicología.

n Estudiantes de la Facultad de Ciencias de la Salud y Trabajo Social, con

experiencias en prácticas vinculadas con la promoción de los derechos de NNA.

También contribuyeron y avalaron el proyecto: el Centro de Extensión Dorrego, el Jardín de Infantes N ${ }^{\circ} 933$, el Programa Primera Infancia del Ministerio de Desarrollo Social de Nación, el Instituto Superior de Formación Docente Manuel Belgrano (ISFDMB), la cátedra Teoría de la Educación de la Licenciatura en Ciencias de la Educación (UNMDP) y el Sindicato Argentino de Docentes Privados (SADOP).

\section{Fundamentos metodológicos y dispositivos propuestos}

Paul Renn (2006) sostiene que en las sociedades modernas la violencia ha tomado proporciones de «epidemia». En el caso particular de la violencia hacia la infancia, las estadísticas ofrecen una clara muestra de la gravedad que representa, dado que implica un impacto traumático en el desarrollo, al tiempo que incrementa la posibilidad de que dichas prácticas se reproduzcan en el futuro. Dado que se trata de un grave problema multicausal, que demanda de urgentes acciones para eliminarlo, resulta operativo considerar diversos aportes de la psicología del desarrollo -especialmente, la teoría del apego y el psicoanálisis vincular-, de las neurociencias y de la psicología sociocomunitaria. Se trata de conceptos que permiten comprender las características del desarrollo infantil y el impacto negativo de las prácticas violentas (muchas de ellas, aún legitimadas socialmente), las causas subyacentes, las consecuencias a nivel psicoafectivo y las posibles acciones para revertirlas. 
En primer lugar, en consonancia con lo planteado, la teoría del apego desarrollada por John Bowlby (1988) resulta de especial interés ya que, junto con los hallazgos de la investigación neurocientífica -así como con otros estudios realizados sobre los efectos del trauma, la regulación de los afectos, la disociación y los tipos de memoria implícitaprocedimental-, permite establecer el papel central que tiene la relación entre cuidador y niñe para la transmisión afectiva y para la regulación emocional; y destaca, además, la importancia de la intersubjetividad en el desarrollo del cerebro y en el dominio cognitivo de la experiencia. Dicha teoría resulta clave para comprender los procesos de subjetivación y el carácter central de los estilos vinculares en el desarrollo personal. En los casos en que los/as padres/madres no se muestran suficientemente disponibles, y no existe una figura de apego sustituta, les niñes pueden verse empujados a adoptar un distanciamiento emocional a través del cual se niega cualquier necesidad de contacto. Si los vínculos de apego han sido seguros en la infancia, la persona encuentra formas de sentir y de expresar el enfado de forma apropiada, sin que la agresividad se desborde y se destruyan las relaciones con otres cuando en dichas relaciones surjan conflictos que desencadenen miedo o pena. En resumen, las formas de cuidado y de apego introducen al niñe en potenciales sendas de desarrollo que conducen a niveles diferentes de adaptación (Renn, 2006).

En segundo lugar, cabe considerar los planteos de las neurociencias, según los cuales el cerebro se desarrolla a través de la experiencia en las primeras etapas de vida. De esta forma, los grupos de neuronas se asocian comunicativamente y se distribuyen funciones cambiantes a través del tiempo. Este paradigma sugiere que el desarrollo tiene «periodos críticos», y que la influencia del entorno familiar y comunitario, así como la calidad de las relaciones afectivas y una sana nutrición en estas etapas de organización funcional, son cruciales en el desarrollo integral del individuo (Aguilar Montiel, 2018).

Finalmente, la psicología comunitaria proporciona herramientas para el trabajo grupal con los diferentes actores involucrados y permite elaborar dispositivos que atiendan a la intervención específica. La intervención comunitaria es entendida como un conjunto de procesos intencionales que se proponen un cambio a través de la participación, de promover el desarrollo de recursos en la población, en las organizaciones comunitarias autónomas, y de fomentar una visión crítica respecto a las representaciones y a los roles en la sociedad (Chinkes, Lapalma \& Niscemboin, 1995, citado por Lapalma, 2001). Este tipo de intervención, fomenta la capacitación y el fortalecimiento de la comunidad, favoreciendo la autogestión para su propia transformación y la de su ambiente, la toma de decisiones y la acción, fortaleciéndose como espacio preventivo (Mori Sánchez, 2008). 
En este aspecto, de acuerdo con Maritza Montero (2011), es importante reafirmar que los cambios en la comunidad no deben promoverse desde el exterior, sino que las transformaciones deben ser efectuadas a partir de la propia comunidad, por esta razón se considera la importancia de otorgar protagonismo a los/as involucrados/as y de ofrecer la oportunidad de generar experiencias que favorezcan la construcción conjunta de criterios y de conceptos referidos a la temática que nos ocupa.

Con respecto a la metodología de trabajo, las acciones requirieron de un abordaje integral a través de diferentes dispositivos de carácter transdisciplinar. Para ello, se necesita de un accionar doble que incluya tanto la acción comunitaria -por ejemplo, a través de la organización de talleres y de espacios de diálogo, y de la difusión de diferentes recursos y materiales de lectura- como la incorporación de esta temática a la formación docente, destinada, especialmente, a les profesionales involucrados en el trabajo con la primera infancia, a fin de promover prácticas respetuosas de los derechos de les niñes como parte de su desarrollo integral.

A través de las tres instituciones participantes, se promovieron prácticas de crianza y de cuidado respetuosas de los derechos de NNA, mediante un abordaje que involucra tanto a les niñes -especialmente, en el proceso de identificación de prácticas vulneradoras y en el desarrollo de recursos de empoderamiento-, como a las familias, les profesionales de salud en ejercicio y en formación, y les docentes en ejercicio y en formación.

\section{Los recursos disponibles}

El presupuesto recibido por la UNMDP para el desarrollo del proyecto fue de 27.000 pesos. Además, se contó con el equipamiento, con las instalaciones y con la infraestructura del Centro de Desarrollo Infantil Jorge Newbery y del Centro de Extensión Universitario (CEU) Dorrego, cuyos espacios físicos resultaron adecuados para el desarrollo de los diferentes talleres.

A esto se sumaron las aulas del Instituto Manuel Belgrano, equipadas para el desarrollo de actividades de capacitación. Las reuniones del equipo ejecutor se desarrollaron en la Facultad de Ciencias de la Salud y Trabajo Social, y en la Facultad de Ciencias Económicas y Sociales. Los cursos de extensión destinados a la formación del equipo y abiertos al público se desarrollaron en el complejo universitario. 


\section{Acciones llevadas a cabo y resultados obtenidos}

A continuación, se detallan las actividades desarrolladas. En todas ellas, se priorizó otorgar protagonismo a les involucrades y ofrecer la oportunidad de generar experiencias que favorezcan la construcción conjunta de criterios y de conceptos referidos a la temática que nos ocupa. Las acciones tuvieron un abordaje integral a través de diferentes dispositivos de carácter transdisciplinar: la acción comunitaria a través de la organización de talleres y la puesta en común de diferentes recursos, la difusión de materiales de lectura informativos y la incorporación de la temática a la formación docente, especialmente, destinada a quienes se desempeñarán con la primera infancia.

1) Jornadas de capacitación interna abiertas al público

El proyecto inició sus actividades con el desarrollo de jornadas de capacitación interna, dos de ellas abiertas al público. La primera, «Infancias y organización social del cuidado. Mar del Plata en el siglo XXI», se desarrolló en dos bloques. Por una parte, se abordó la necesidad de profundizar en la diversidad de situaciones socioeconómicas en las que se desarrollan las crianzas, puesto que la situación laboral y material de las familias constituye un condicionante en el ejercicio del cuidado. Las familias cuentan con escasos dispositivos institucionales de sostén de la crianza, y en un contexto de deterioro socioeconómico las posibilidades de resolver necesidades de nutrición, salud, educación y cuidado se ven sensiblemente afectadas. Las carencias repercuten en el bienestar de NNA y en los sujetos que desarrollan estas tareas, mayoritariamente mujeres. Tales condiciones de vida ponen en jaque la noción misma de niñez, en tanto que el propio derecho a transitar dicha etapa del ciclo vital en su totalidad se ve vulnerado por la temprana exposición al trabajo remunerado y no remunerado, y por la necesidad de sumarse o, incluso, de generar estrategias de subsistencia y de organización de la vida doméstica.

Por otra parte, se expusieron las desigualdades interseccionales en los mundos del trabajo remunerado y no remunerado, los alcances y las limitaciones de las políticas públicas de cuidado, así como la sobrecarga de tareas y los mandatos sociales que recaen sobre las mujeres-madres. Las instituciones de cuidado gratuitas son casi inexistentes y los permisos laborales remunerados para el cuidado, exiguos y desiguales entre varones y mujeres, lo cual redunda en que las mujeres-madres sean las únicas depositarias de la responsabilidad social del cuidado. A partir de la clasificación de modelos de cuidado propuesta por Arlie Hochschild (2008), se compartieron fragmentos de videos que fueron utilizados como disparadores para pensar la diversidad de situaciones en las que se produce el cuidado y la diversidad de vulnerabilidades a las que están expuestos les niñes. 
Finalmente, se presentaron algunas claves para el análisis crítico de los discursos sobre crianza desde una perspectiva interseccional y de derechos humanos. En esta jornada participaron 25 personas, incluido el equipo del proyecto. La capacitación permitió poner en común saberes de las diversas disciplinas que confluían en el proyecto y elaborar los consensos básicos sobre los cuales se construía la propuesta.

La segunda jornada, «Trabajo emocional, control emocional y construcción de las subjetividades en el siglo XXI. Discursos hacia la primera infancia en la educación y la literatura», contó con la participación de 12 personas, incluido el equipo del proyecto. El taller permitió la aproximación al material bibliográfico utilizado en los talleres de reflexión-capacitación docente, y los usos y las apropiaciones en el marco de las transformaciones del Diseño Curricular para el nivel inicial 2019, que recupera y propone una implementación de la «educación emocional» abordada desde las propuestas del proyecto como «tecnologías del yo» que procuran una gestión individual de las emociones y una compartimentación de lo emocional como contenido, sin llegar a poner en tensión las dinámicas institucionales y vinculares presentes en el ámbito educativo.

La propuesta de trabajo del taller se desarrolló, en primer lugar, en torno al análisis del concepto de «trabajo emocional», con la intencionalidad de vincular esta noción con la tarea desempeñada por les docentes que se dedican a trabajar con la primera infancia. En segundo lugar, se propuso el trabajo con diferentes textos literarios mediante los cuales se planteó el modo en el que a través de estos discursos se construye una determinada imagen de la infancia, así como del vínculo entre adultes y niñes. Esta actividad tuvo como objetivo realizar un trabajo de meta análisis con el fin de observar las posibles formas de abordar la conducta infantil, especialmente cuando es disruptiva. En este sentido, se establece una distinción entre prácticas que consideran a les niñes como sujetos de derecho y aquellas que evidencian el modo en que la infancia es representada en condición de subalternidad, con la prevalencia de una mirada negativa que trae aparejada por parte de les adultes actitudes de carácter punitivo.

\section{2) Reunión interproyectos}

Con la intención de iniciar la construcción de dispositivos reflexivos para el análisis de las prácticas de crianza, se desarrollaron reuniones internas en las que se trabajó junto con el equipo del proyecto de extensión «Voces del Caribe», correspondiente a la Escuela de Medicina de la UNMDP. En ese espacio se intercambiaron materiales bibliográficos y marcos conceptuales que permitieron delinear los materiales gráficos para pensar la crianza. 
3) Reuniones para ampliar la base territorial del proyecto

En el espacio del CEU Dorrego, donde se radicó el proyecto, se inició el vínculo con el Programa Primera Infancia, de la Secretaría de Niñez del Ministerio de Desarrollo Social de la Nación, para abordar los alcances territoriales y temáticos del programa, conocer el material formativo y acordar actividades en común.

4) Taller de reflexión y de capacitación docente

En el marco de una jornada obligatoria de capacitación docente, en el Jardín de Infantes N. ${ }^{\circ} 933$ se desarrolló, con todo el equipo docente de la institución, el taller «Trabajo emocional, control emocional y construcción de las subjetividades en el siglo XXI. Discursos hacia la primera infancia en la educación y la literatura». Se compartieron investigaciones precedentes -incluidas algunas de integrantes del equipo- sobre el trabajo emocional y las representaciones de la docencia en el nivel inicial, desde una perspectiva de género.

Se compartieron diversas obras literarias para niñes organizadas en tres grupos: vínculos, emociones y experiencias. Estos materiales constituyeron disparadores para pensar las representaciones de les niñes, así como las apropiaciones y los usos teleológicos de la literatura como mecanismo disciplinador. Asimismo, se habilitó un espacio de reflexión para analizar los lineamientos políticos en los que se inscribe la «educación emocional» y los posicionamientos ideológicos con los que dialoga. En este sentido, el trabajo del proyecto ha promovido actividades de investigación.

El taller de capacitación involucró a 22 docentes de la institución, quienes expresaron interés y compromiso por el desarrollo de la propuesta. Resultó fructífero el intercambio y la diversidad de pareceres respecto de la incorporación del concepto de «educación emocional». Se observaron tanto recepciones muy favorables como resistencias vinculadas con las divergencias entre la propuesta del taller y el enfoque prescripto en el Diseño Curricular para la Educación Inicial de la provincia de Buenos Aires, que prevé una implementación más que un análisis crítico por parte de les docentes (Corral, 2012). Asimismo, algunas de las integrantes del equipo se encontraban asistiendo a la capacitación oficial sobre educación emocional, lo cual resultó valioso para aproximarse a las expectativas de ese documento y, al mismo tiempo, para profundizar la posible tensión con una mirada crítica-analítica. Se acordó continuar avanzando en actividades con docentes y con familias de la comunidad educativa. 
5) Taller con facilitadoras del Programa Primera Infancia

En las sedes de Islas Malvinas y Félix U. Camet, se llevó a cabo el taller «Desafíos de la crianza en la primera infancia. Recursos para pensar vínculos y prácticas». El taller se desarrolló en dos turnos y espacios, que son de encuentro semanal entre los equipos de facilitadoras. Se propuso la lectura de dos ficciones para niñes en las que una adulta -madre, en ambos casos- marca una pauta de conducta frente a una acción inadecuada del niñe protagonista de la historia. A partir de ella, se ofrecían una serie de preguntas de reflexión para pensar, a partir de los vínculos desarrollados entre los personajes, las prácticas de crianza y las representaciones sobre les niñes que aparecían en las historias. Finalmente, se intercambiaban semejanzas y divergencias entre las prácticas de crianza observadas por las facilitadoras y las representadas en las historias. Algunas facilitadoras compartieron la persistencia de una mirada adultocéntrica de la niñez, mientras que otras observaban indicios de transformación en las prácticas y en las representaciones.

El taller alcanzó a 40 facilitadoras y fue acompañado por las profesionales del equipo técnico, con quienes luego se mantuvo un encuentro de evaluación de la experiencia. Surgió del intercambio la inquietud de desarrollar herramientas capaces de conciliar la eficacia comunicativa y la diversidad cultural e interseccional, y de construir, al mismo tiempo, una representación del sujeto niñe respetuosa de sus derechos, de su autonomía y de las particulares necesidades de esa etapa del ciclo vital.

6) Taller

En el Instituto Superior de Formación Docente Manuel Belgrano (ISFDMB), la cátedra Teoría de la Educación de la Licenciatura en Ciencias de la Educación (UNMDP) y el Sindicato Argentino de Docentes Privados (SADOP) desarrollaron el taller «Pensar la "educación emocional": subjetividades, docencia y niñez en el siglo XXI», que recuperó y complejizó los lineamientos propuestos por el desarrollado en el Jardín de Infantes N. ${ }^{\circ}$ 933. El taller alcanzó a 20 estudiantes de los profesorados en Educación Inicial y Educación Primaria (ISFDMB), 80 estudiantes de la Licenciatura en Ciencias de la Educación, y 70 docentes y estudiantes de carreras docentes en SADOP. En los tres casos, se produjo una recepción positiva de las dinámicas y los debates propuestos, así como de las tensiones entre la apuesta a revisar los procesos de subalternización de les niñes y las violencias y los privilegios que es necesario cuestionar y modificar desde los roles adultos en las instituciones y en los espacios públicos y domésticos. Quedaron inquietudes e intereses que permitirán avanzar en nuevas propuestas de trabajo. 
7) Difusión de la propuesta

Las integrantes del equipo fueron invitadas por diversos programas radiales locales para compartir la propuesta del proyecto de extensión. Allí describieron algunos de los abordajes realizados en las actividades y contextualizaron la propuesta en las representaciones y los vínculos que se establecen con les niñes, desde una perspectiva interseccional.

En septiembre de 2019, el equipo presentó el proyecto en las VII Jornadas de Extensión y Compromiso Social «Mariano Salgado», organizadas por la Secretaría de Extensión de la UNMDP, mediante la exposición de un poster y la participación en un conversatorio. ${ }^{3}$

8) Diseño de recursos para operadores comunitarios

Para pensar las propias prácticas y los discursos populares y profesionales sobre las prácticas de crianza, se desarrolló el dispositivo denominado «Consejos vendo... Recursos para construir una mirada analítica sobre los discursos y los mandatos sociales vinculados a la crianza» [Figura 1]. El objetivo fue proponer una labor reflexiva antes que prescriptiva, que permita a cada sujeto en situación de cuidado/crianza construir prácticas autónomas y autogestionadas, puesto que el primer paso para promover la autonomía y la autodeterminación de les niñes es favorecer la construcción de formas de criar asentadas en decisiones y elecciones reflexivas, y no en la obediencia a autoridades/mandatos sociales. El dispositivo quedó accesible para su aplicación por parte de facilitadoras, docentes y otros agentes comunitarios vinculados con las prácticas de crianza.
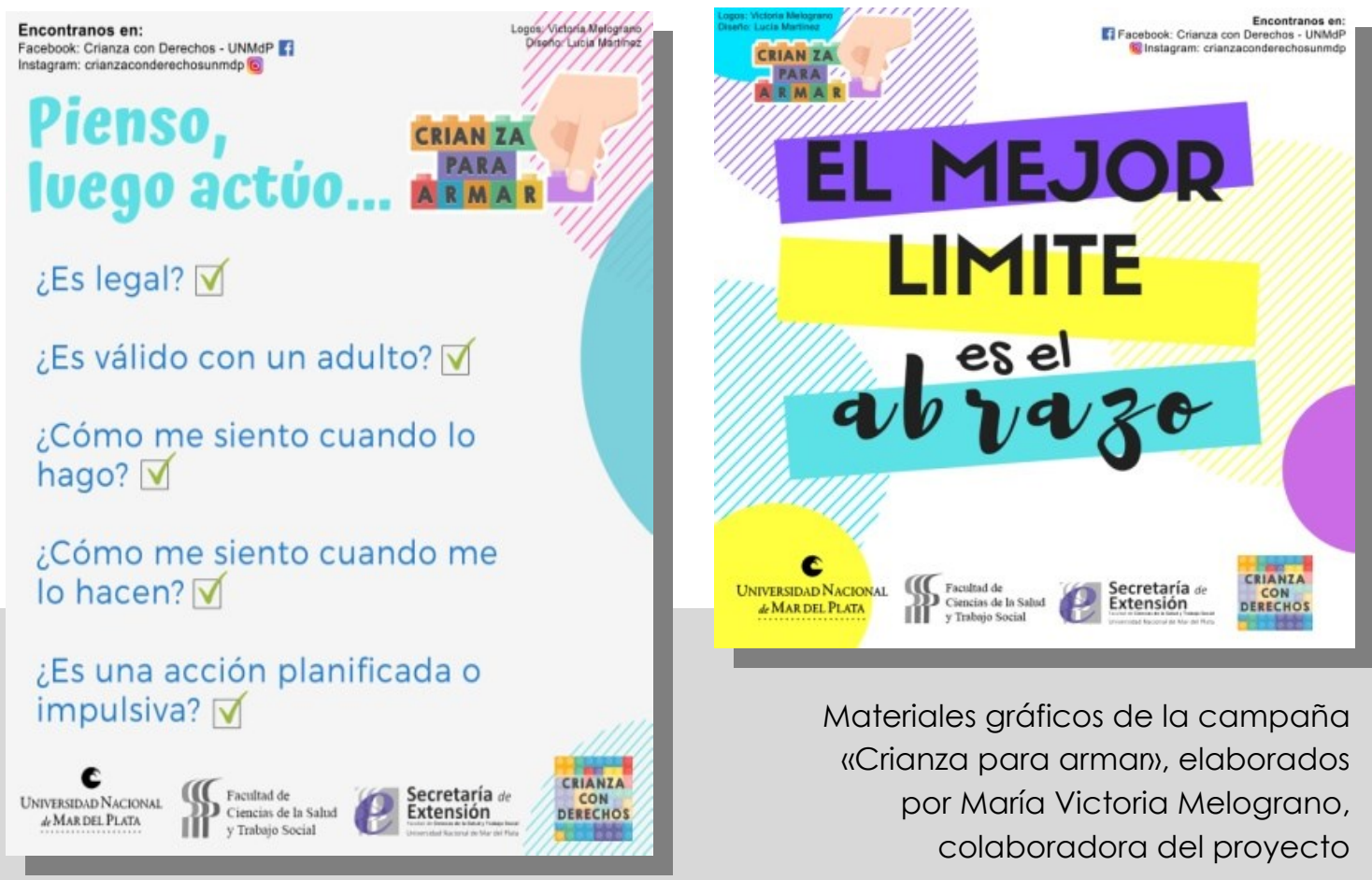

Materiales gráficos de la campaña "Crianza para arman», elaborados por María Victoria Melograno, colaboradora del proyecto 
9) Diseño de una campaña gráfica interactiva

Para la reflexión sobre los discursos y las prácticas de crianza se llevó a cabo la campaña gráfica interactiva «Crianza para armar». Esta actividad, que supuso el cierre de la primera etapa del proyecto, incluyó el diseño y la difusión de tres materiales gráficos destinados a promover una mirada amable y respetuosa hacia les niñes, así como una serie de preguntas que permiten interpelar sobre las prácticas y los discursos de crianza ajenos y propios para la construcción de prácticas respetuosas de la diversidad y con perspectiva interseccional.

Su distribución se realizó por diversos canales, siendo el epicentro el CEU Dorrego, desde donde se promovió su acceso en el Centro de Salud N. ${ }^{\circ}$ 1, El Servicio Social Oeste, el Centro de Promoción y Protección de Derechos de NNA y las instituciones de nivel inicial y primario del área de influencia del CEU, con el Jardín de Infantes $\mathrm{N}^{\circ} 933$ como primera institución involucrada.

Al cierre de cada encuentro, se relevó el nivel de satisfacción y de adecuación de los contenido trabajados, así como nuevas inquietudes que, desde el punto de vista de les participantes, ameriten generar nuevos espacios de intercambio

El proyecto transitó su primera fase durante 2019, y desde entonces logró avanzar en el establecimiento de intereses y de diagnósticos comunes con las instituciones vinculadas, además de ampliar la base territorial que había sido delineada al inicio, para lo cual la radicación en el CEU tuvo un rol fundamental. Desde el establecimiento de un equipo de trabajo nuevo -con lazos entre algunos/as de sus integrantes en virtud de diversas actividades en común-, se construyó un grupo con una formación y con objetivos comunes y con capacidades acumuladas para continuar avanzando en la extensión vinculada a las prácticas de crianza, desde una perspectiva interseccional y de derechos humanos.

Los vínculos con los/as destinatarios/as también comenzaron a partir de la implementación del proyecto. En todos los casos, los lazos establecidos permitieron avanzar en el trabajo conjunto. El proyecto ha llegado, en el transcurso del año, a un cierre de actividades con una campaña de impacto y potencialidad de réplica y trascendencia.

Se concretó la articulación con instituciones y con sectores del medio en la utilización de recursos y en el desarrollo de estrategias comunes en torno a las problemáticas abordadas. El equipo de trabajo pudo construir, gracias al intercambio con el territorio, 
y a partir de la integración de diversos saberes disciplinares vinculados con el género, les niñes, la crianza y el cuidado, una mirada integral en la que confluyeron las ciencias sociales y de la salud para la construcción colectiva de una «crianza crítica» (Cutuli, 2020). El proyecto, junto a una comunidad protagonista, impulsó un proceso de transformación social colectivo cuyo modo de vincularse se aleja de las prescripciones y las vigilancias institucionales hegemónicas en los discursos sobre crianza, con particular intensidad en los sectores socioeconómicos más vulnerados.

En este sentido, se recuperaron saberes interdisciplinarios que permiten pensar la provisoriedad y los campos de poder que implican los consensos científicos, además de la adopción de roles de autoridad desde los cuales se comunican valores y creencias no necesariamente vinculados al saber y al rol profesional que se detenta. De este modo, los diálogos construidos no compiten ni reemplazan las intervenciones profesionales específicas que acompañan el desarrollo del niñe sano. Se propone la construcción de herramientas junto con las personas que transitan la crianza, útiles para pensar en las propias prácticas así como en los discursos que se les imponen, muchos de ellos desde los roles profesionales.

En sus inicios, la propuesta de «Crianza con derechos» recuperó experiencias precedentes de las actividades de investigación y de extensión de algunas integrantes del equipo de trabajo. Su desarrollo permitió repensar y ampliar los alcances de la propuesta original, dando lugar a diversas posibilidades de integralidad que se fortalecieron a partir del proyecto. Una de las líneas que se profundizó, vinculada a la mirada crítica sobre la implementación de la «educación emocional», promovió el análisis de los textos producidos y apropiados para su implementación en el aula. Las herramientas comunicativas y el avance en la línea de talleres con docentes han sentado bases para la elaboración de algunos productos de transferencia: materiales para la reflexión sobre la crianza y los trayectos formativos para docentes de nivel inicial y primario sobre el abordaje interseccional y de derechos humanos de la crianza, la educación y el cuidado.

Asimismo, se valoran los actores que contribuyeron con el proyecto y con la construcción de nuevos conocimientos: el CEU Dorrego, el Jardín de Infantes N. ${ }^{\circ} 933$, el Programa Primera Infancia, el Instituto Superior de Formación Docente Manuel Belgrano (ISFDMB), la cátedra Teoría de la Educación de la Licenciatura en Ciencias de la Educación (UNMDP) y el Sindicato Argentino de Docentes Privados (SADOP). 


\section{Reflexiones}

El proyecto promueve la reflexión con diversos actores territoriales sobre los discursos y las prácticas de crianza a partir del desarrollo de talleres de reflexión, de dispositivos y de material gráfico destinado al análisis antes que a la prescripción, para familias y para actores territoriales que acompañan la crianza. Las prácticas de violencias naturalizadas así como las representaciones negativas sobre el sujeto niñe se pusieron de manifiesto en el proceso de implementación, tanto a través de la puesta en común de experiencias de intervención por parte de los actores territoriales como de las valoraciones en primera persona.

En este sentido, algunos de los recursos propuestos, como el uso de historias ficcionales, permitieron un diálogo más abierto y despersonalizado sobre los vínculos entre adultes y niñes, así como la revisión sobre la internalización y la legitimidad de las prácticas adultocéntricas, aún de aquellas que reconocen la subalternidad de las mujeres-madres en tanto cuidadoras pero no de les niñes como sujetos en situación de vulnerabilidad. Los espacios de reflexión junto con la comunidad promovieron una mirada de les niñes orientada a traducir esta vulnerabilidad en cuidados.

Al interior de la universidad, la incorporación de les niñes como sujetos sociales implica un desafío en proceso para las diversas áreas de acción y para la revisión de las propias prácticas institucionales en una institución que se asume como integrada exclusivamente por adultes, pero que es siempre atravesada por niñes a través de los vínculos de cuidado de trabajadores universitarios, de docentes y de estudiantes. Un desafío pendiente para «Crianza con derechos» implica, entonces, la promoción de esta reflexión en diversas áreas de la vida universitaria. La incorporación del sujeto niñe en las actividades docentes y de investigación de las integrantes del equipo constituye un aporte en esta línea que es recuperado por la directora y la codirectora del proyecto.

El proyecto se realizó sin una de las organizaciones convocadas inicialmente, el CAPS $\mathrm{N} .^{\circ} 2$, por dificultades institucionales. De todas maneras, la multiplicidad de actores involucrados, lo que incluyó las actividades de promoción de la salud que se desarrollan en el Programa Primera Infancia y en los Servicios locales de Promoción y Protección de Derechos de Niños, Niñas y Adolescentes del Partido de General Pueyrredon, permitieron compensar dicho inconveniente. Los talleres territoriales con familias han sido acordados con el Jardín Provincial N. 933 para su desarrollo en 2020,4 dados los tiempos institucionales requeridos por las autorizaciones que permitan su implementación. 
Las actividades de sistematización, así como el desarrollo de dispositivos territoriales, la campaña gráfica y las posibilidades de transferencia emergidas del proyecto, promueven la trascendencia temporal y territorial de la propuesta. Asimismo, la formación y la experiencia apropiadas por estudiantes favorece, en el largo plazo, la continuidad de la línea de extensión sobre niñez, interseccionalidad y derechos humanos en la que se inscribe el proyecto.

El desarrollo de espacios de comunicación y de intercambio, en los que se promueva la escucha profesional y el diálogo libre de juicios de valor, produce lazos de confianza entre las comunidades y les profesionales. Desde estos espacios de diálogo se construyeron, fruto de la interacción entre la propuesta del proyecto «Crianza con derechos» y las inquietudes manifestadas por los actores participantes, herramientas reflexivas y formativas para familias y para docentes, acerca de los valores-guía que acompañan los procesos de cuidado y de crianza.

Asimismo, el desarrollo de las actividades de intervención tiene la potencialidad de instalar la temática en la agenda de la comunidad, lo que promueve el intercambio y la reflexión más allá de las actividades propuestas por el proyecto. La necesidad de prácticas de cuidado y de crianza respetuosas de los derechos de les niñes constituye un valor en sí mismo, cuyo impacto en la cotidianeidad es inmediato.

Asimismo, la incorporación de les niñes como sujetos de cuidado constituye una deuda del campo de estudios vinculado a la organización social del cuidado, construido en torno a la legítima preocupación por las desigualdades en la distribución social y sexual de las tareas de cuidado. La «crisis de los cuidados» (Pérez Orosco, 2006; Ezquerra, 2010) constituye un fenómeno que requiere de un abordaje de género para no reproducir desventajas económicas, laborales y sociales para las mujeres-madres. El cuidado respetuoso debe ser reivindicado como un derecho humano inalienable, independientemente de su funcionalidad económica y política en la reproducción del sistema económico y social.

Las actividades desarrolladas en el marco del proyecto enriquecen los saberes en este campo de estudios, que ofrece un relevante foco de interés tanto para el Grupo de Estudios del Trabajo de la Facultad de Ciencias Económicas y Sociales (UNMDP), al cual pertenecen la directora y codirectora del proyecto, como para la Facultad de Ciencias de la Salud y la Escuela de Medicina, donde se fortalece el desarrollo del conocimiento en estas áreas a partir de la formación interna y de las prácticas sociocomunitarias. En interacción con las experiencias vehiculizadas por el proyecto de extensión, es posible promover el fortalecimiento de los saberes docentes a través de la formación de grado y posgrado, 
y de talleres de capacitación interna para docentes de la Licenciatura en Trabajo Social. En la formación de grado, la organización social del cuidado con perspectiva de género y centrada en los derechos constituye un aporte del que las estudiantes que integran el proyecto serán las primeras involucradas, pero que repercute enriqueciendo la formación de todes les estudiantes, a través de la transversalización del tema y la introducción de asignaturas específicas.

Desde el equipo de trabajo del proyecto se sostiene la relevancia del cuidado y la crianza en virtud de los derechos de les niñes y no como una mera apuesta al futuro adulto. La niñez constituye por sí misma una etapa valiosa de la vida, que si bien resulta de vital importancia para la constitución de la vida adulta, no es solo en función del futuro ciudadano-productor-consumidor que la niñez importa. En este sentido, si bien desde las acciones propositivas que involucran a les niñes se construyen mejoras hacia el futuro, estas tienen un valor intrínseco a partir de la transformación de la cotidianeidad de cada niñe que recibe el trato, la contención y la guía adecuada a sus derechos y sus necesidades.

Agradecimientos: a Romina Cutuli, Valeria Melczarski, Paola Cabero, Carolina Calienes, Yesica Celiberti, Mercedes Santarone, Lorena Basilico, Eva Martin, Ingrid Aliaga y Lucía Martínez, sin cuya dedicación y expertise el desarrollo de esta experiencia no hubiera sido posible. También a las siguientes instituciones: CEU Dorrego de la UNMDP, Programa Primera Infancia del Ministerio de Desarrollo Social de Nación, Instituto Superior de Formación Docente Manuel Belgrano (ISFDMB), cátedra Teoría de la Educación de la Licenciatura en Ciencias de la Educación (UNMDP) y Sindicato Argentino de Docentes Privados (SADOP).

\section{Referencias}

Aguilar Montiel, B. (2018). El aporte de las neurociencias para una educación temprana de calidad. RELAdEI. Revista Latinoamericana de Educación Infantil, 7(1), 98-100. Recuperado de http://www.usc.es/revistas/index.php/reladei/article/view/5274/0

Alegre, P. y otros (2011). Diagnóstico de la situación socioproductiva del Partido de General Pueyrredon. Grupos vulnerables y políticas de cohesión. Proyecto UNMdP -MGPEU-LA-WIN European Union and Latin America for Welfare INTEGRATEDPOLICIES. Mar del Plata, Argentina: UNMdP. 
Aspiazu, E. y Cutuli, R. (2015). Políticas de cuidado infantil en Argentina. Aportes para su clasificación y evaluación. En E. Lanari y C. Hasanbegovic (Comps.), Mujeres latinoamericanas. El presente en veintidós letras (pp. 339-371). Mar del Plata, Argentina: Eudem.

Bowlby, J. (1988). Una base segura. Aplicaciones clínicas de una Teoría del Apego. Barcelona, España: Paidós.

Cabero, P. (2018). Violencia familiar. Propuestas para su visibilización. Un estudio desde el Equipo de Gestión Social Territorial Oeste de la ciudad de Mar del Plata. 2015/2016 (Trabajo de especialización). Facultad de Ciencias de la Salud y Trabajo Social, Universidad Nacional de Mar del Plata, Argentina.

Carli, S. (2002). Niñez, pedagogía y política. Transformaciones de los discursos acerca de la infancia en la historia de la educación argentina entre 1880 y 1955. Ciudad Autónoma de Buenos Aires, Argentina: Miño y Dávila.

Corral, A. (coord.) (2012). Diseño Curricular para la Educación Inicial. Primer ciclo. La Plata, Argentina: Dirección General de Cultura y Educación de la Provincia de Buenos Aires. Recuperado de http://servicios2.abc.gov.ar/lainstitucion/organismos/consejogeneral/diseni oscurriculares/documentosdescarga/dc_con_resolucion.pdf

Cutuli, R. (2020). Crianza con Derechos. Enlace Universitario, (34), 5. Recuperado de https://www3.mdp.edu.ar/attachments/article/68/Enlace34.pdf

Donzelot, J. (1979). La policía de las familias. Valencia, España: Pre-Textos.

Ezquerra, S. (2010). La crisis de los cuidados: orígenes, falsas soluciones y posibles oportunidades. Viento Sur, (108), 37-43. Recuperado de https://vientosur.info/la-crisis-de-los-cuidados-origenes-falsas-soluciones-yposibles-oportunidades/ 
Forero-Londoño, O. F. (2011). La violencia escolar como régimen de visibilidad. Magis. Revista Internacional de Investigación en Educación, 4(8). Recuperado de http://revistas.javeriana.edu.co/index.php/MAGIS/article/downlo $\operatorname{ad} / 3571 / 2686$

Hochschild, A. (2008). La mercantilización de la vida íntima.

Ciudad Autónoma de Buenos Aires, Argentina: Katz.

Instituto Nacional de Estadística y Censos (INDEC) (2018).

Trabajo e ingresos. Mercado de trabajo. Indicadores socioeconómicos (EPH). Cuarto trimestre de 2017. Informes Técnicos, vol. 2, núm. 56. Recuperado de

https://www.indec.gob.ar/uploads/informesdeprensa/indicadores_ eph_4trim17.pdf

Lapalma, A. (2001). El escenario de la intervención comunitaria. Revista de Psicología de la Universidad de Chile, 10(2), 61-70. Recuperado de https://revistapsicologia.uchile.cl/index.php/RDP/article/view/18573

Llobet, V. (Coord.) (2014). Pensar la infancia desde América Latina. Un estado de la cuestión. Ciudad Autónoma de Buenos Aires, Argentina: Consejo Latinoamericano de Ciencias Sociales (CLACSO).

Maté, V. (2017). Aproximación diagnóstica. Centro de Atención Primaria de la Salud «Alto Camet». Mar del Plata, Argentina: Municipalidad de General Pueyrredon.

Ministerio de Desarrollo Social de la Nación y UNICEF (2013).

Encuesta

sobre Condiciones de Vida de Niñez y Adolescencia. Principales Resultados, 2011-2012. Recuperado de https://bit.ly/346ec1s

Montero, M. (2011). Introducción a la psicología comunitaria.

Ciudad Autónoma de Buenos Aires, Argentina: Paidós. 
Mori Sánchez, M. D. P. (2008). Una propuesta metodológica para la intervención comunitaria. Liberabit, 14(14), 81-90. Recuperado de https://dialnet.unirioja.es/descarga/articulo/2766779.pdf

Nari, M. (2004). Políticas de maternidad y maternalismo político. Buenos Aires, 1890-1940. Ciudad Autónoma de Buenos Aires, Argentina: Biblos.

Pérez Orozco, A. P. (2006). Amenaza tormenta: la crisis de los cuidados y la reorganización del sistema económico. Revista de economía crítica, (5), 7-37. Recuperado de http://revistaeconomiacritica.org/sites/default/files/revistas/n5/1_amenaza _tormenta.pdf

Renn, P. (2006). Apego, trauma y violencia. Comprendiendo las tendencias destructivas desde la perspectiva de la teoría del apego. En C. Harding (Ed.), Aggression and Destructiveness: Pyschoanalytic Perspectives (pp. 59-78). Nueva York, Estados Unidos: Routledge.

Rustoyburu, C. (2012). Infancia y maternidad en los discursos de la pediatría psicosomática (Buenos Aires, a mediados del siglo XX). Tempos Históricos, 16(1), 59-86.

Sagua, M. C y Sabuda, F. G. (2015). ¿Territorios jóvenes en una comuna envejecida a nivel poblacional? Las recientes dinámicas de crecimiento demográfico asociadas al hábitat en la ciudad de Mar del Plata y el Partido de General Pueyrredon. 1991-2001-2010. En P. I. Lucero (Ed.), Atlas de Mar del Plata y el partido de General Pueyrredon II: problemáticas socio-territoriales contemporáneas (pp. 23-50). Mar del Plata, Argentina: EUDEM.

Theidon, K. (2004). Entre prójimos. Estudio sobre la salud mental y la violencia política en Ayacucho. Lima, Perú: Instituto de Estudios Peruanos (IEP). 


\section{Notas}

1 De este $10 \%$, alrededor de un tercio se encuentra en esa situación desde hace más de un año (INDEC, 2018).

2 Lamentablemente, desde fines de 2018 y durante gran parte de 2019, el CAPS se enfrentó a dificultades institucionales que lo mantuvieron en inactividad. Esto generó la desvinculación institucional del referente con quien se había acordado la colaboración y llevó al avance del proyecto sin esa organización. En este mismo sentido se proponía el desarrollo de actividades en el Centro de Desarrollo Infantil.

3 En octubre, la directora presentó el libro infantil Mientras todos duermen (Metrópolis, 2019), de Romina Cutuli y María Laura Kuin, un relato ilustrado con historias pensadas para hablar con niñes sobre el mundo del trabajo -que impacta en las propias situaciones de crianza-, en las calles, en los hogares y en las aulas, lo cual se enmarca en los objetivos de este proyecto.

4. Debido al Aislamiento Social Preventivo y Obligatorio (ASPO), decretado por el gobierno nacional el 20 de marzo de 2020 ante la pandemia por Covid-19, estas actividades debieron replantearse, generándose instancias de comunicación virtuales y haciendo uso de diferentes redes sociales. 